\title{
Pre-action games \& exercises via tele-rehabilitation: A prototype for supplemental stroke therapy
}

\author{
Vince Macri, James Merrill*, Paul Zilber, Vanessa Lane and William Quinn \\ NeuroJungle, LLC 434 Lacy Woods Tallahassee, FL 32312, USA
}

\begin{abstract}
We describe Pre-Action Games \& Exercises (PAGEs) a prototype for supplemental tele-rehabilitation. PAGEs are therapist-guided exercises for chronically impaired stroke survivors: basically a computer-based platform of brain exercises based on control of virtual limbs. Survivors control virtual limbs, simultaneously actuating wearable, motorized orthoses which physically manipulate impaired limbs. Physical manipulations mirror survivor controlled virtual limb movements. PAGEs are designed to assist physical and occupational therapists to treat increased numbers of stroke survivors (estimated, in the US, Canada, Mexico and Europe to be 18 million individuals). Key elements of PAGEs tele-rehabilitation are outlined. Tele-rehabilitation is suggested as a modality for therapist-supervised, survivor-selfadministered and repetitive brain-to-body exercises either in clinics and/or at home or anywhere at any time.Thus, the genetically determined pace of reduction of the primary venous plexuses determines the final shape of the dural venous system and its compensatory abilities.
\end{abstract}

\section{Introduction}

PAGES are designed to be a supplemental tool for therapists. Advances in pre-hospital, specialized mobile unit medical diagnosis and treatment, i.e. onboard first responder interventions, result in more stroke cases treated sooner and with positive results. For example, in cases of ischemic stroke, onboard administration of tPA (Tissue-Type Plasminogen Activator) is, in some cases, practiced as pre-hospital thrombolytic therapy. ${ }^{1}$ It is saving lives and improving outcomes. A new, May 2018, US Food and Drug Administration approved technology enables onboard diagnosis of severe strokes. It may be used by mobile unit paramedics. ${ }^{2}$ The technology combines transcranial ultrasound with an artificial intelligence (AI) component. AI algorithms compare on-board patient findings with thousands of stored images of severe stroke survivors to help diagnose the current survivor's condition: therefore a pre-hospital early alert to neurosurgeons. In addition, inhospital endovascular surgical advances ${ }^{3}$ to 'coil-out' blood clots and other medical and surgical advances have increased survival rates and work-loads carried by physical and occupational therapists.

\section{Background}

In almost all countries, therapist-to-patient ratios are daunting. In any country, if one divides the total number of chronically impaired stroke survivors by the total number of physical and occupational therapists, the quotient is a significantly high ratio of patients per therapist. For example, since therapy is usually beneficial at a rate of three sessions per week and adding hours for therapists' documentation, patient preparation and discharge per week for each patient, the sum of hours overwhelms therapists' reasonable number of weekly work hours.

${ }^{1}$ The Cleveland Clinic Mobile Stroke Unit (https://my.clevelandclinic.org/health/ treatments/17242-mobile-stroke-unit) administers tPA.

${ }^{2} \mathrm{FDA}$ approved AI powered diagnostic tools in 2018 (https://www.technologyreview. com/f/610853/fda-approves-first-ai-powered-diagnostic-that-doesnt-need-a-doctors-help/).

${ }^{3}$ Endovascular surgery can be used as a stroke prevention measure (https://utswmed.org/ conditions-treatments/endovascular-surgery/).
Therapists' workloads burden the costs associated with stroke. In 2008 US stroke survivor related costs were estimated to be more than $\$ 65$ billion per year [1]

\section{Method}

Among stroke survivors, hemiparesis affects as many as $50 \%$ of individuals age 65 and older [2]. Given that hemiparesis entirely or substantially immobilizes movements of physical limbs, PAGEs virtual limb technology presents substitutes for moving impaired limbs. PAGEs presents survivors with anatomically realistic virtual limbs controlled within true range of motion. Virtual limbs enable survivors to create simulated physical movements relevant to impaired limb rehabilitation. In that sense, PAGEs closely mirror conventional hands-on and assisted movement techniques used by therapists to rehabilitate physically impaired limbs (Figure 1).

Operationally, a stroke survivor uses an unimpaired hand and a standard computer mouse (or other input means) to point and drag a virtual limb (or any part thereof) so as to simulate unimpaired virtual movements. The process reinforces each survivor's innate ability for motor imagery i.e. the mental abstraction of visualizing movements before actually making physical movements. Motor imagery a/k/a motor visualization is an effective process used for years by athletes, some individuals in stroke rehabilitation and multiple kinds of simulations used in training. Motor imagery is a preview to each survivor's actual movement. PAGEs make it possible for survivors to instantiate the abstraction of purposeful movements into viewable representations of movements and to receive virtual visuo-motor feedback from simulated physical movements. In effect PAGEs represents brain exercises of personal movement purpose.

${ }^{*}$ Correspondence to: James Merrill, NeuroJungle, LLC 434 Lacy Woods Tallahassee, FL 32312, USA, Tel: 978-764-4295; E-mail: james@neurojungle.com

Received: October 28, 2019; Accepted: November 13, 2019; Published: November 18, 2019 


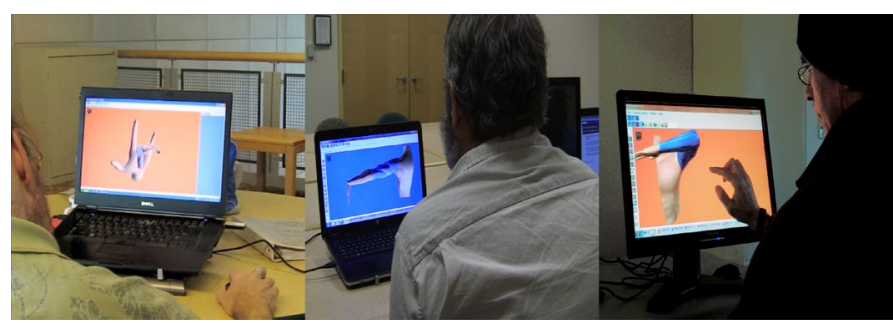

Figure 1. PAGEs closely mirror conventional hands-on and assisted movement techniques used by therapists to rehabilitate physically impaired limbs

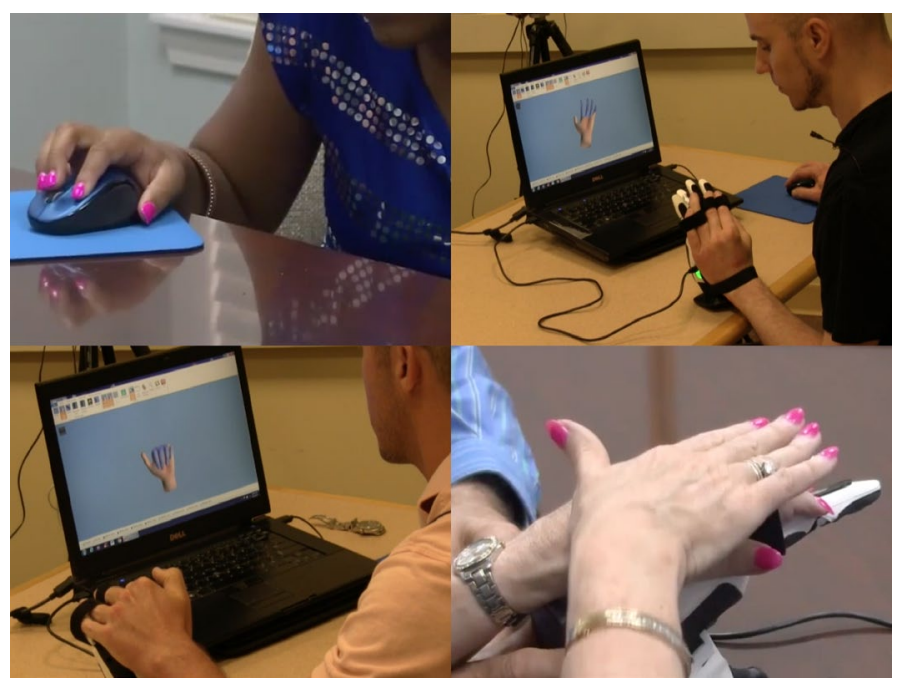

Figure 2. PAGEs foster physical exercise of impaired limbs

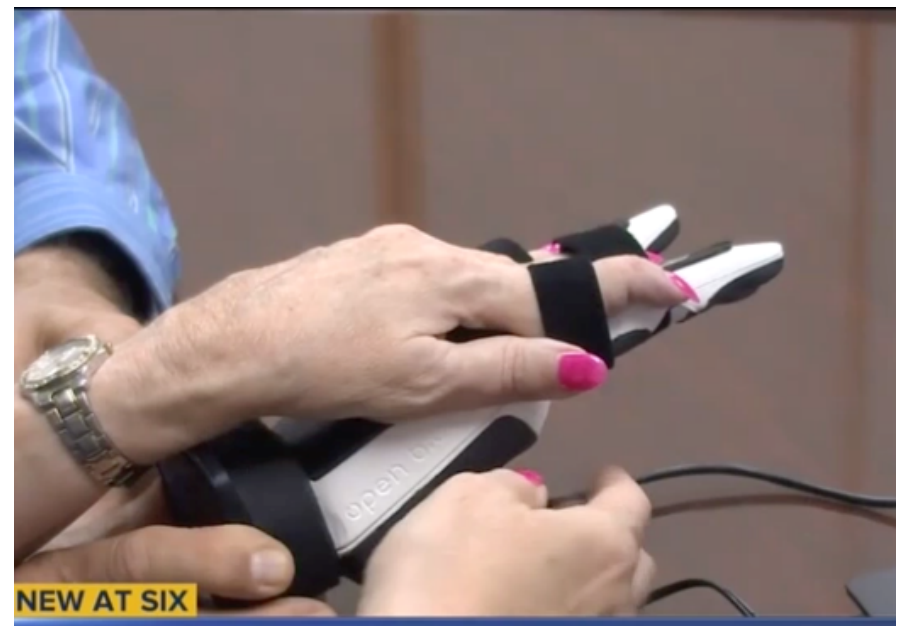

GAMING FOR STROKE SURVIVORS

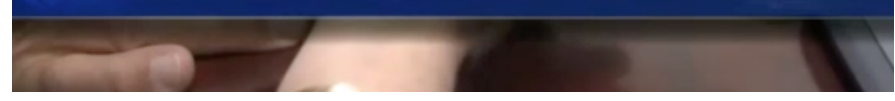

Figure 3. The PAGEs system signals a motorized orthotic device, attached to the impaired limb, to mirror the actions of the survivor-driven virtual limb

PAGEs have physiological antecedents. Published research shows viewing and interacting with images of motion and limbs [3] and mental training can improve movement outcomes [4] and the human brain plans movements before the body makes physical movements [5].

In addition to brain exercises, PAGEs foster physical exercise of impaired limbs. For example, when using PAGEs three interconnected events can take place: 1 - a virtual limb is survivor-controlled to simulate movements of the physically impaired limb; 2 - the PAGEs system signals a motorized orthotic device, attached to the impaired limb, to mirror the actions of the survivor-driven virtual limb; and 3 -the wearable devices (powered orthoses) exercise actual, physically impaired limbs (Figures 2 and 3).

\section{Discussion}

PAGEs and motorized orthoses, i.e. wearable limb exercise devices, leverage neuroplasticity. Survivors' brain functions plan movements before the body makes them. Brain planning processes are supported by the phenomena of mental immersion in virtual environments. PAGEs, presented in virtual environments, aid survivors to re-learn to move impaired limbs before limbs can be moved.

Implications of hemiparesis include the inability to move impaired or weakened limbs. Almost all cases present without external injury to the limbs e.g. there are no cuts, bruises, burns and/or abrasions affecting intact, but impaired limbs. Almost all therapy is directed to physical manipulation of intact limbs, which may be impaired, in part, by disuse and are therefore candidates for physical manipulation(s). Therapists either practice hands-on manipulation or use assistive technology, or both. More recently, forms of virtual reality (VR) have been added to rehabilitation protocols. Most VR applications require $1^{\text {st }}$. person physical, patient movements displayed in virtual environments: however, moving impaired limbs is neither easy or, in many cases, possible for patients with hemiparesis.

Physical and occupational therapy techniques are numerous: soft tissue mobilization; strain-counter-strain; joint mobilization; muscle energy techniques; high velocity low amplitude thrusting; and any number of pain reduction techniques. Few, if any, directly emphasize brain-to body signaling exercises. For tele-rehabilitation to be usefully supplemental it should enable chronically impaired stroke survivors to engage in brain-to-body exercises and device-assisted physical movements of impaired limbs which track conventional rehabilitation techniques. Numerous uses of VR in rehabilitation involve patients actually, physically moving. The movements are displayed in a virtual environment, which is to say displayed actions are $1^{\text {st }}$ person. PAGEs however, enables survivors to control virtual limbs and author simulated physical movements, i.e. $3^{\text {rd }}$ person actions, which are distinctly brain exercises. In that the location of stroke damage is the brain PAGEs are direct and efficient survivor rehabilitation. PAGEs support brain planning for physical movements: for example please see below, "dynamic internal image[s] of anticipated actions" and "brain mechanisms that create internal motor representations of the action, that is to say cortical simulation s....."

Regarding brain processes before physical movements are made, please see Ramachandran and Iacoboni: "When I ask you to salute, you conjure up a visual image of the salute and, in a sense, use the image to guide your arm movements." "[the]... supramarginal gyrus, is very much involved in this ability to create a dynamic internal image of anticipated actions...." Ramachandran [6] and "... motor planning for physical actions is generated by brain mechanisms that create internal motor representations of the action, that is to say cortical simulations...." [7].

VR is uniquely suited to immerse stroke survivors in an environment which captures imagination, instantiates motor imagery, invokes feelings of presence and ownership and give survivors a sense of agency, i.e. that virtual limbs are personal, controllable agents and extensions of their own body parts. As such controlling virtual limbs can be ordered, observed, evaluated and prescribed via tele-rehabilitation. 
Studies on VR body illusions are numerous, extensive and scientifically sound. We suggest as quite elegant, precise and informative the study, referenced below, by Slater and Sanchez-Vives [8].

\section{References}

1. Di Carlo Antonio (2009) Human and economic burden of stroke, Age and Ageing 38: 4-5.

2. Roger VL, Go AS, Lloyd-Jones DM, Benjamin EJ, Berry JD, et al. (2012) Heart disease and stroke statistics--2012 update: a report from the American Heart Association. Circulation 125: e2-20.

3. Polli A, Moseley GL, Gioia E, Beames T, Baba A, et al. (2017) Graded motor imagery for patients with stroke: a non-randomized controlled trial of a new approach. Eur $J$ Phys Rehabil Med 53: 14-23.
4. Binder E, Hagelweide K, Wang LE, Kornysheva K, Grefkes C, et al. (2014) Sensory-guided motor tasks benefit from mental training based on serial prediction. Neuropsychologia 54: 18-27.

5. Wilhelm Klaus (2007) "How the brain moves." MaxPlanckResearch pp: 33-36

6. Ramachandran VS (2012) The Tell-Tale Brain: A Neuroscientist's Quest for What Makes Us Human. W.W. Norton \& Company, New York.

7. Iacoboni M, Woods RP, Brass M, Bekkering H, Mazziotta JC, et al. (1999) Cortical mechanisms of human imitation. Science 286: 2526-2528.

8. Slater M, Spanlang B, Sanchez-Vives MV, Blanke O (2010) First person experience of body transfer in virtual reality. PloS one 5: e10564.

Copyright: @2019 Macri V. This is an open-access article distributed under the terms of the Creative Commons Attribution License, which permits unrestricted use, distribution, and reproduction in any medium, provided the original author and source are credited. 